\title{
Standards und Richtlinien für Hospiz- und Palliativversorgung in Europa: Teil $\mathbf{1}^{1}$ Weißbuch zu Empfehlungen der Europäischen Gesellschaft für Palliative Care (EAPC)
}

\author{
White Paper on Standards and Norms for Hospice and Palliative Care in Europe: Part 1 \\ Recommendations of the European Association for Palliative Care
}

Autoren

Institute
L. Radbruch ${ }^{1}$, S. Payne ${ }^{2}$

Übersetzt von D. Büche, E. Schmidlin, S. Jünger

European Association for Palliative Care (EAPC) President

2 EAPC Vice-President

und der EAPC-Vorstand (Michaela Bercovitch, Augusto Caraceni, Tine De Vlieger, Pam Firth, Katalin Hegedus, Maria Nabal, André Rhebergen, Esther Schmidlin, Per Sjøgren, Carol Tishelman, Chantal Wood, and Honorary Director Franco De Conno)

\author{
Schlüsselwörter \\ - Standards \\ - Normen \\ - Qualität \\ - Delphi Prozedur \\ - European Association \\ for Palliative Care \\ Keywords \\ - standards \\ - norms \\ - quality \\ - Delphi procedure \\ - European Association \\ for Palliative Care
}

\section{Zusammenfassung}

$\nabla$

Die Europäische Gesellschaft für Palliative Care (EAPC) legt mit diesem Weißbuch Standards und Normen für Hospize und Palliative Care in Europa vor. Der Inhalt richtet sich an die Fachgesellschaften und das Personal, die direkt mit der Palliativversorgung zu tun haben. Selbstverständlich spielen - neben der allgemeinen Struktur der Versorgung - auch andere Merkmale bei der Qualität der Versorgung von Schwerkranken und Sterbenden eine wichtige Rolle. Dazu gehören u.a. Behandlungsmethoden, Qualitätsmessungen, Therapieerfolge, Forschung, Bildung, Finanzierung, Verfahrensweisen und Organisation sowie Gesetzgebungen. Mit der vorliegenden Richtlinie, die in einem Konsens mit den europäischen Fachgesellschaften formuliert wurden, kann die EAPC Empfehlungen für einen einheitlichen Qualitätsstandard geben. Themenrelevante Inhalte werden in einer Reihe zukünftiger Publikationen detaillierter behandelt.

\section{Bibliografie}

DOI http://dx.doi.org/

10.1055/s-0031-1276909

Z Palliativmed 2011; 12 :

216-227

(c) Georg Thieme Verlag KG Stuttgart · New York .

ISSN 1615-2921

\section{Korrespondenzadresse} Univ.-Prof. Dr. Lukas Radbruch European Association for Palliative Care (EAPC), Klinik für Palliativmedizin, Universitätsklinikum Bonn

Tel.: +49-228-287-13495

Zentrum Palliativmedizin, Malteser Krankenhaus Bonn/ Rhein-Sieg

Von-Hompesch-Straße 1

53123 Bonn

Tel.: +49-228-64819170

Fax: +49-228-64819209

lukas.radbruch@malteser.org

\section{Hintergrund}

$\nabla$

Die moderne Hospizbewegung begann 1967 mit der Eröffnung des St. Christopher's Hospice in London durch Cicely Saunders. Schon 1975 sah sich Balfour Mount mit einer Begriffsproblematik konfrontiert, als er eine Bezeichnung für seine neue Abteilung im Spital Montreal suchte, denn im französischsprachigen Kanada war der Begriff „Hospiz“ bereits anderweitig besetzt, was ihn veranlasste, den Begriff „Palliative Care“ einzuführen.

\footnotetext{
1 Translated from the article „White Paper on standards and norms for hospice and palliative care in Europe: part 1" first published in the European Journal of Palliative Care 2009; 16 (6): 278-289 and 2010; 17 (1): 22-33. Kindly reproduced by permission of the publishers of the EJPC, who retain the copyright. All rights reserved. www. ejpc.eu.com
}

\section{Abstract \\ $\nabla$}

The focus of this White Paper is on norms and standards for hospice and palliative care in Europe as recommended by the EAPC. In addition to the structure of care, many other dimensions play an important role in the provision of quality hospice and palliative care - such as treatment methods, quality and outcome measurement, research, education, funding, policy and organisation, and legislation. The White Paper presents a common terminology and standards that have been formulated in a consensus procedure with the European palliative care associations. Relevant topics will be examined in more depth in a series of future papers.
Seither geht das Ringen um einen einheitlichen Sprachgebrauch in der Hospiz- und Palliativversorgung weltweit weiter, was auch die Entwicklung von internationalen Standards und Richtlinien behindert. Unlängst gab die Europäische Gesellschaft für Palliative Care (EAPC) eine Bestandsaufnahme zur Entwicklung der Palliativversorgung in den europäischen Ländern in Auftrag. Die Arbeitsgruppe wurde von Carlos Centeno und David Clark geleitet und hat nun ihre Ergebnisse im EAPC Atlas for Palliative Care herausgegeben, der zum ersten Mal valide Ver-

Übersetzung des Artikels „White Paper on standards and norms for hospice and palliative care in Europe: part 1“. Erstpublikation im European Journal of Palliative Care 2009; 16 (6): 278-289 and 2010; 17 (1): 22-33. Mit freundlicher Genehmigung der Herausgeber der EJPC, die das Copyright behalten. Alle Rechte vorbehalten. www. ejpc.eu.com 
gleichsdaten zum Stand der Palliativversorgung in den europäischen Ländern liefert [1,2]. Die Auswertung zeigte einige Gemeinsamkeiten, aber auch eine große Variabilität bezüglich der Strukturen der Versorgungsangebote. Diese Unterschiede sind zumindest teilweise bedingt durch ein unterschiedliches Verständnis der zugrunde liegenden Konzepte und Begriffe der Palliativversorgung. Die Entwicklung einer gemeinsamen Terminologie wurde als Voraussetzung für aussagekräftige Vergleiche gefordert [3].

Diese Situation veranlasste die EAPC, nun auf der Grundlage eines Konsensverfahrens mit den nationalen Fachgesellschaften eine einheitliche europäische Terminologie vorzuschlagen. Die Richtlinien basieren auf diesen im Konsens definierten Fachbegriffen. Eine Orientierungshilfe durch Richtlinien und Standards ist nicht nur für diejenigen notwendig, die professionell in der Hospiz- und Palliativversorgung tätig sind, sondern auch für die Entscheidungsträger im Gesundheitswesen, die dafür verantwortlich sind, allen Patienten einen adäquaten Zugang zur Palliativversorgung zu ermöglichen.

Mit der fortschreitenden Entwicklung der Palliativversorgung in ganz Europa stehen die Verantwortlichen nicht nur vor der Entscheidung, wo und wann Angebote der Palliativversorgung entwickelt werden sollen, sondern auch, wie diese umzusetzen und zu gestalten sind. Eine adäquate Strukturqualität ist Voraussetzung für eine hohe Versorgungsqualität. Behörden und politische Entscheidungsträger achten auf Kosteneffizienz und versuchen, die Kosten durch eine Minimierung von Personal und Kostenerstattung zu reduzieren. Die Pflegeorganisationen kämpfen um adäquate Personalressourcen, die für eine hochqualitative Versorgung notwendig sind. In diesem Konflikt brauchen beide Parteien Empfehlungen bezüglich der erforderlichen Strukturqualität.

In diesem Positionspapier legt die EAPC Richtlinien für die Strukturqualität der Palliativversorgung im stationären und ambulanten Sektor im Rahmen verschiedener Versorgungskonzepte vor. Das Weißbuch berücksichtigt dabei die unterschiedlichen Konzepte der verschiedenen europäischen Länder und Regionen. Aus diesem Grund wurden Richtlinien und keine Standards definiert. Während Standards eine absolute Grenze festlegen, unterhalb der qualitativ gute Palliativversorgung nicht möglich ist (Minimalstandards), stellen Richtlinien einen Konsens von Qualitätszielen dar, die angestrebt werden sollten. Werden diese Richtwerte erreicht, ist mit Sicherheit von einer hohen Qualität auszugehen.

\section{Methoden}

\section{$\nabla$}

Dieses Weißbuch wurde entwickelt, um im Gesundheitssystem Tätigen und Entscheidungsträgern eine Orientierungshilfe und Empfehlungen an die Hand zu geben. Der erste Entwurf wurde im Februar 2008 im Rahmen einer Krebskonferenz der Europäischen Union unter der slowenischen Präsidentschaft vorgestellt. Wie eingangs dargelegt, will die EAPC eher Richtlinien und Arbeitsdefinitionen als Standards vorlegen. Standards definieren starre Grenzen mit Cut-off-Werten, was bedeuten würde, dass Versorgungseinheiten, die diese Standards nicht erreichen, den Spezialistenstatus verlieren würden. Die EAPC-Richtlinien legen fest, wie eine hochqualitative Palliativversorgung aussehen sollte, aber Versorgungsangebote, die - bedingt durch lokale oder regionale Unterschiede - eine oder mehrere dieser Richtlinien nicht erfüllen, sollen aufgrund dessen nicht diskriminiert wer- den. In Anbetracht der unterschiedlichen Gesundheitssysteme wie auch der unterschiedlichen kulturellen Hintergründe scheint es kaum möglich, mit den nationalen Gesellschaften von über 20 Ländern Standards zu vereinbaren, wohingegen ein Konsens bezüglich Richtlinien machbar erscheint.

Der Vorstand der EAPC beauftragte eine Arbeitsgruppe (bestehend aus Saskia Jünger, Sheila Payne und Lukas Radbruch), dieses Papier vorzubereiten. Der Manuskriptentwurf wurde von Experten der EAPC (Franco De Conno, Carl-Johan Fürst, Geoffrey Hanks, Irene Higgingson, Stein Kaasa, Phil Larkin, Friedemann Nauck) gegengelesen. Nach den Rückmeldungen der Experten wurde der Entwurf überarbeitet und den Vorstandsmitgliedern der nationalen Mitgliedsgesellschaften der EAPC vorgelegt. Die Stellungnahmen der nationalen Gesellschaften wurden mithilfe der Delphi-Methode eingeholt, d.h. es wurde nach dem Ausmaß der Zustimmung oder Ablehnung bezüglich der einzelnen Richtlinien gefragt. Vertreter von 35 verschiedenen nationalen Hospiz- und Palliativgesellschaften aus 22 europäischen Ländern nahmen an dem Konsensverfahren teil. Auf der Grundlage der Rückmeldungen aus dem Delphi-Verfahren wurde die abschließende Version des Papiers vorbereitet. Diese endgültige Version wurde dem Vorstand der EAPC vorgelegt und als offizielles Positionspapier der EAPC angenommen.

\subsection{Ziel der Richtlinien}

Richtlinien können auf nationaler und regionaler/lokaler Ebene entwickelt werden. Auf nationaler Ebene verkörpern sie eine einheitliche Strategie für die weitere Entwicklung und Kontrolle, die Zeit und Energie sparen hilft. Auf regionaler oder lokaler Ebene werden die nationalen Richtlinien den spezifischen Charakteristika der entsprechenden Regionen oder Institutionen angepasst. Eine Kombination beider Verfahren stellt sicherlich die beste Vorgehensweise dar [4]. Die Einführung von Richtlinien setzt eine weitreichende fachliche Abstimmung sowie das Einverständnis aller beteiligten Teilnehmer voraus, damit Validität und Relevanz der Leitlinien sichergestellt sind. Zweck und Ziele der Richtlinien zur Palliativversorgung sind in $\bullet$ Tab. 1 beschrieben.

\footnotetext{
Tab. 1 Zweck / Ziele der Richtlinien zur Palliativversorgung (Quelle: [5]).

Fördern der Qualität und Verringern der Unterschiede in neuen und bestehenden Programmen

Entwicklung und Unterstützung der Versorgungskontinuität über verschiedene Versorgungsstrukturen hinweg

Fördern der Zusammenarbeit und Partnerschaft zwischen Palliativversorgungsprogrammen, Gemeindehospizen und einem breiten Spektrum anderer Anbieter der Gesundheitsversorgung

Fördern der Entwicklung und der kontinuierlichen Verbesserung aller klinischen Programme der Palliativversorgung

Festlegen einheitlich akzeptierter Definitionen der wichtigsten Kernelemente der Palliativversorgung, die eine gleichbleibende Qualität und Zuverlässigkeit der betreffenden Versorgungsangebote sicherstellen Festlegen von nationalen Zielsetzungen für den Zugang zu hochqualitativer Palliativversorgung

Fördern von Initiativen zur Leistungserfassung und Qualitätsentwicklung
}

\subsection{Kernbereiche der Richtlinien zur Palliativversorgung} Dieses Papier basiert nicht auf einer systematischen Literaturübersicht, sondern auf Publikationen und anderen Quellen anerkannter offizieller Institutionen wie z.B. dem Europarat. Die Kernaussagen dieser Veröffentlichung wurden von bestehenden Leitlinien und Empfehlungen zur Palliativversorgung unter- 
schiedlicher Organisationen, Fachgesellschaften, Nationen und Komitees abgeleitet [4-7]. Das Papier befasst sich mit folgenden Kernbereichen der Palliativversorgung:

- Definition und Terminologie der Hospiz- und Palliativversorgung

- gemeinsame Werte und Philosophie

- Ebenen der Versorgung

- Patientengruppen

- Versorgungsangebote und Versorgungsstrukturen

Andere Kernbereiche wie die Verfügbarkeit und der Zugang zu Versorgungsangeboten, Aus-, Fort- und Weiterbildung für im Gesundheitswesen Tätige, klinische Zertifizierungen und Dokumentationsstandards, Öffentlichkeitsarbeit sowie Forschung werden nicht hier, sondern separat abgehandelt.

\subsection{Problemfelder}

Das Konsensverfahren für Richtlinien für eine qualitativ hochstehende Palliativversorgung in der Europäischen Union birgt aufgrund der beträchtlichen Heterogenität der Versorgungsstrukturen in den verschiedenen Ländern einige Herausforderungen. Einige Arten von Versorgungsangeboten existieren nur in bestimmten Ländern [1]. Beispielsweise haben die Ergebnisse der Untersuchung durch die EAPC Task Force gezeigt, dass unter den 52 europäischen Ländern nur 5 Länder ein gut ausgebautes und flächendeckendes Netzwerk für stationäre Hospize haben. Dagegen verfügen andere Länder über einen viel höheren Anteil an ambulanten Palliativ-Care-Teams. Dies führt zu der Frage, ob ein gemeinsames Konzept den verschiedenen regionalen, geografischen und kulturellen Rahmenbedingungen für die Palliativversorgung gerecht werden kann. Das Gleiche gilt für Begriffe und Definitionen. Hier könnte sich die Aufgabe, einen Konsens zu erzielen, noch schwieriger gestalten als in Nordamerika. Schließlich besteht die Europäische Union aus Ländern mit einer jahrtausendealten Geschichte und verschiedenen Sprache, die sie von- einander trennen, und es muss berücksichtigt werden, dass die Länder stolz sind auf diese Unterschiede [3].

Im Rahmen eines Konsensverfahrens muss daher auch geprüft werden, inwieweit die europäischen Richtlinien zur Palliativversorgung mit den jeweiligen demografischen und kulturellen Aspekten der verschiedenen Länder und Regionen in Einklang gebracht werden können.

Die Politik der Palliativversorgung ist ein Gebiet, das sich enorm schnell entwickelt; seit dem Beginn des europäischen Konsensverfahrens wurden 2 wichtige Berichte von Regierungen publiziert. Im Juli 2008 wurde vom Department of Health in England die „End of Life Care Strategy“ veröffentlicht [8]; im April 2009 wurde die zweite Auflage der Konsensrichtlinien des National Consensus Project der USA herausgegeben [9]. Sowohl die Neuerungen dieser zweiten Auflage als auch die spezifischen Richtlinien der „End of Life Care Strategy“ konnten beim europäischen Konsensverfahren nicht berücksichtigt werden. Im Sinne einer Post-hoc-Validierung hat das Lenkungskomitee sichergestellt, dass die Aussagen dieses Weißbuches noch mit den Empfehlungen dieser neuesten Dokumente übereinstimmen.

\section{Fachbegriffe / Terminologie}

Das Vorhaben, die Hospiz- und Palliativversorgung in Europa zu untersuchen und zu vergleichen, brachte eine heterogene Terminologie zutage $[1,10]$. Die Unterschiede zeigten sich nicht nur in der wissenschaftlichen Literatur, sondern auch in Gesetzestexten, Regierungsbeschlüssen und Stellungnahmen von Experten relevanter Fachgesellschaften [10]. Zum Beispiel fanden Pastrana et al. im Rahmen einer Diskursanalyse von Definitionen in der Palliativversorgung den Gebrauch unterschiedlicher Begriffe für sich überschneidende medizinische Bereiche wie „Hospice“, „Hospice Care“, „Continuing Caring“, „End-of-Life Care“, „Thana-

\begin{tabular}{|c|c|c|}
\hline Land & Begriff für „Palliative Care“ & andere Begriffe \\
\hline Österreich & $\begin{array}{l}\text { Palliativ(e) Care; Palliativmedizin; palliative Betreuung } \\
\text { / Palliativbetreuung; Palliativversorgung }\end{array}$ & lindernde Fürsorge \\
\hline Belgien (flämisch) & Palliatieve zorg & \\
\hline Belgien (wallonisch) & Soins palliatifs & \\
\hline $\begin{array}{l}\text { Kroatien (Republik } \\
\text { Kroatien) }\end{array}$ & Paljativna skrb & \\
\hline Zypern & Anakoufistiki frontitha / frontida & Paragoritikes agogis \\
\hline Tschechien & Paliativni pece & \\
\hline Dänemark & Palliative indsats; palliative behandeling (og pleje) & \\
\hline Frankreich & Soins Palliatifs & Accomagnement de fin de vie \\
\hline Deutschland & Palliativmedizin; Palliativversorgung & (ehrenamtliche) Hospizarbeit \\
\hline Griechenland & Anakoufistiki frontida & \\
\hline Ungarn & Palliativ ellts & \\
\hline Island & Liknarmedferd/liknandi medferd & \\
\hline Irland & Palliative Care & \\
\hline Israel & Tipul tomeh / tomech; tipul paliativi & Palliative care \\
\hline Italien & Cure palliative & \\
\hline Lettland & Paliativa aprupe & \\
\hline Niederlande & Palliaitieve zorg & Terminale zorg (Terminal Care) \\
\hline Norwegen & $\begin{array}{l}\text { Palliativ behandeling; palliativ omsorg; palliativ madi- } \\
\text { sin; palliativ pleie }\end{array}$ & $\begin{array}{l}\text { Lindrende behandling og om- } \\
\text { sorg; palliative care }\end{array}$ \\
\hline Portugal & Cuidados paliativos & \\
\hline Spanien & Cuidados Paliativos & \\
\hline Schweden & Palliativ vard; palliative medicin & \\
\hline Schweiz & Palliative Care; palliative Betreuung & \\
\hline UK & Palliative care; palliative medicine & $\begin{array}{l}\text { Supportive and palliative care; } \\
\text { end-of-life care }\end{array}$ \\
\hline
\end{tabular}

Tab. 2 Gebräuchliche Begriffe für „Palliative Care“ in verschiedenen europäischen Ländern. 
tology“, „Comfort Care“, „Supportive Care“ [11]. Überdies können verschiedene Begrifflichkeiten auf verschiedene kulturelle Hintergründe zurückgeführt werden. In der deutschen Sprache gibt es z.B. kein Äquivalent für den Ausdruck „Palliative Care“ und folglich wird der Ausdruck „Palliativmedizin“ sowohl für „Palliative Care“ als auch für „Palliative Medicine“ verwendet [11]. Allerdings hat der synonyme Gebrauch von „Palliativmedizin“ (Palliative Medicine) und Palliative Care in diesem Zusammenhang zu Bedenken hinsichtlich einer Medikalisierung der Palliativversorgung in Deutschland geführt [11]. Deshalb verwenden einige Experten den englischen Begriff „Palliative Care“, um ihn von Palliativmedizin zu unterscheiden. Erst in jüngerer Zeit hat sich in Deutschland auch der Begriff „Palliativversorgung“ durchgesetzt ${ }^{2}$. Ähnliches gilt für andere europäische Sprachen, bei denen es nicht klar ist, inwieweit mit dem offiziellen Begriff „Palliative Care" $(\bullet$ Tab.2) die gleichen Konzepte gemeint sind.

Es ist offensichtlich, dass ein effektiver europäischer Ansatz zur Qualitätsförderung der Palliativversorgung einen eindeutigen Gebrauch der Begriffe erfordert, was ein gegenseitiges Einvernehmen bezüglich der Definition dieser Begriffe voraussetzt. Die Task Force zur Entwicklung der europäischen Palliativversorgung hat die Entwicklung und Verfügbarkeit von Versorgungsangeboten in 42 europäischen Ländern im Atlas of Palliative Care verglichen [2]. In einer kritischen Bewertung des Atlas wurde die Relevanz einer gemeinsamen Sprache unterstrichen, denn vergleichende Darstellungen können nur dann einen Einfluss haben, wenn sie auf einer gemeinsamen Sprache basieren, in der jedes Wort in jedem Land, in dem es verwendet wird, die gleiche Bedeutung hat [3].

Da ein absoluter Konsens in Europa unter Berücksichtigung der Sprachen und Kulturen gegenwärtig nicht möglich ist, wird die EAPC die nachfolgenden Arbeitsdefinitionen für Hospiz- und Palliativversorgung gebrauchen.

Im folgenden Text heben die fettgedruckten Textpassagen die Empfehlungen der EAPC hervor.

\subsection{Palliativversorgung}

Palliativversorgung ist die aktive, umfassende Versorgung des Patienten, dessen Erkrankung auf eine kurative Behandlung nicht anspricht. Kontrolle von Schmerzen, anderen Symptomen sowie von sozialen, psychologischen und spirituellen Problemen ist oberstes Ziel. Palliativversorgung ist interdisziplinär in ihrem Ansatz und umfasst den Patienten, die Familie und deren Umfeld. Palliativversorgung stellt somit das grundlegendste Konzept der Versorgung dar - dasjenige, das sich an den Bedürfnissen des Patienten orientiert, wo immer er oder sie betreut wird, sei es zu Hause oder im Krankenhaus. Palliativversorgung bejaht das Leben und sieht das Sterben als normalen Prozess; weder beschleunigt noch verhindert sie den Tod. Sie strebt danach, die bestmögliche Lebensqualität bis zum Tod zu erhalten [12].

Trotz der kulturellen Unterschiede bezüglich des palliativen Versorgungsansatzes in Europa hat die EAPC-Definition der Palliativversorgung einen vereinenden Einfluss auf die Palliative-CareBewegung und Organisationen in vielen europäischen Ländern [13]. Die EAPC-Definition unterscheidet sich minimal von derjenigen der WHO (World Health Organisation), die nachfolgend aufgeführt ist.

Palliativversorgung ist ein Ansatz, der die Lebensqualität von Patienten und deren Familien verbessert, die mit Problemen im Zu-

\footnotetext{
2 In dieser Übersetzung wird der Begriff „Palliativversorgung“ als Übersetzung für „Palliative Care“ verwendet.
}

sammenhang mit einer lebensbedrohenden Erkrankung konfrontiert sind, und zwar mittels Prävention und Linderung von Leiden durch frühzeitiges Erkennen und umfassende Erfassung sowie durch die Behandlung von Schmerz und anderen Problemen im körperlichen, psychosozialen und spirituellen Bereich [14].

Diese WHO-Definition hat die ältere WHO-Definition ersetzt, die sich auf Patienten beschränkte, deren Erkrankung nicht auf kurative Behandlung anspricht [15]. Die neue und modifizierte WHODefinition von 2002 erweiterte den Blickwinkel der Palliativversorgung auf Patienten und deren Familien, die mit Problemen im Zusammenhang mit einer lebensbedrohenden Erkrankung konfrontiert sind [16].

\subsection{Hospizversorgung}

Hospizversorgung betrachtet eine Person ganzheitlich mit dem Ziel, allen Bedürfnissen - körperlichen, emotionalen, sozialen und spirituellen - gerecht zu werden. Betreut werden Patienten am Ende ihres Lebens sowie deren Angehörige - zu Hause, in Tageskliniken und in stationären Hospizen. Das Personal sowie Ehrenamtliche arbeiten in multiprofessionellen Teams zusammen, um eine Betreuung zu bieten, die sich an den individuellen Bedürfnissen und Entscheidungen orientiert und hierbei Schmerzfreiheit, Würde, Frieden und Ruhe anstrebt [17].

Die Definition der Hospizversorgung findet deutlich weniger Konsens als die Definition der Palliativversorgung. Es scheint grundlegende Unterschiede im Verständnis der Hospizversorgung zu geben, die möglicherweise die verschiedenen Arten der Nutzung von Hospizen in Westeuropa widerspiegeln. In einigen Ländern gibt es einen klaren Unterschied zwischen Hospizversorgung und Palliativversorgung, während in anderen Ländern die Begriffe synonym verwendet werden. Wenn ein Unterschied gemacht wird, kann dieser mit institutionellen Rahmenbedingungen zusammenhängen, bei denen eine Palliativstation als Abteilung innerhalb eines Krankenhauses gilt, während ein stationäres Hospiz ein eigenständiges Versorgungsangebot darstellt. Die Unterscheidung kann jedoch auch durch den Einweisungsgrund oder die Art der angebotenen Interventionen begründet sein. Unter diesem Aspekt ist in Deutschland z.B. eine Palliativstation ein Teil eines Krankenhauses, der sich in erster Linie der Krisenintervention und medizinischen Stabilisierung widmet, während ein stationäres Hospiz Sterbebegleitung für Patienten anbietet, die nicht zu Hause betreut werden können. Diese Kategorisierung wird in anderen Ländern so nicht verwendet.

In einigen Ländern gibt es ähnliche Unterscheidungen für ambulante Versorgungsangebote. In Deutschland ist die ambulante Palliativversorgung auf die Pflege fokussiert, während der ambulante Hospizdienst vor allem psychosoziale Unterstützung durch Ehrenamtliche anbietet.

In einigen Ländern hat der Begriff Hospiz eher die Bedeutung einer Philosophie der Betreuung als einer bestimmten Struktur, in der Versorgung angeboten wird [18]. Zum Beispiel steht in Deutschland Hospizarbeit vor allem für Betreuungsleistungen, die ihre Wurzeln in einer Bürgerbewegung haben und stark auf ehrenamtlichem Engagement basieren, wohingegen Palliativversorgung - und noch spezifischer Palliativmedizin - als medizinischer Fachbereich angesehen wird.

Da sich jedoch sowohl die zugrunde liegende Philosophie als auch die Definitionen von Hospiz- und Palliativversorgung in weiten Teilen überschneiden, steht der Begriff Palliativversorgung in diesem Papier sowohl für Hospiz- als auch für Palliativversorgung. 


\subsection{Supportive Care}

Supportive Care bezeichnet die Vorsorge und das Management der Beeinträchtigungen durch eine Krebserkrankung und deren Behandlung. Dies beinhaltet körperliche und psychosoziale Symptome sowie Nebenwirkungen unter Berücksichtigung des kompletten Erlebens einer Krebserkrankung, was auch die Verbesserung der Rehabilitation und der Lebensbedingungen nach Überleben einer Krebserkrankung umfasst [19]. Es besteht eine beträchtliche Überschneidung und keine klare Trennung zwischen dem Gebrauch der Begriffe „Palliativversorgung“ (Palliative Care) und „Supportive Care“.

Die meisten Experten stimmen darin überein, dass mittels Supportive Care diejenigen Patienten betreut werden sollten, die noch unter antineoplastischer Therapie stehen bzw. auch Patienten einbezogen werden sollten, die eine Krebserkrankung überlebt haben, während der Schwerpunkt der Palliativversorgung auf der Betreuung von Patienten mit weit fortgeschrittenen Erkrankungen liegt, bei denen antineoplastische Therapien beendet wurden [20]. Hingegen hat eine nationale deutsche Untersuchung gezeigt, dass 9\% der Patienten auf Palliativstationen Chemotherapie erhalten [21]. Überlebende einer Krebserkrankung werden dagegen nicht in die Zielgruppe der Palliativversorgung eingeschlossen. Andererseits befasst sich Palliativversorgung nicht nur mit Tumorpatienten, sondern auch mit Patientengruppen mit anderen lebensbedrohlichen Erkrankungen.

Supportive Care sollte nicht als Synonym für Palliativversorgung verwendet werden. Supportive Care ist ein Teil der Onkologie, während die Palliativversorgung ein eigenes Fachgebiet darstellt, das sich auf alle Menschen mit einer lebensbedrohlichen Erkrankung bezieht.

\subsection{Versorgung am Lebensende (End-of-Life Care)}

Versorgung am Lebensende kann synonym für Palliativversorgung und Hospizversorgung verwendet werden, wobei das Lebensende als Zeitrahmen von 1-2 Jahren verstanden wird, währenddem der Patient, die Familie und die Behandler realisieren, dass die Erkrankung lebensbegrenzend geworden ist.

Der Begriff „Versorgung am Lebensende“ findet in Nordamerika breite Verwendung und wurde in europäischen Ländern vom Gesetzgeber aufgegriffen - gelegentlich mit dem Verständnis, dass Palliativversorgung vorwiegend mit Tumorpatienten in Verbindung gebracht wird, wohingegen „Versorgung am Lebensende“ für alle Patienten verwendbar ist. In England z. B. wurde im Sommer 2008 eine „NHS End of Life Strategy“ publiziert, um das Sterben aller Patienten, wo auch immer sie betreut werden, zu verbessern [8].

„Versorgung am Lebensende“ kann auch spezifischer aufgefasst werden als umfassende Betreuung für sterbende Patienten in den letzten Stunden oder Tagen ihres Lebens.

Versorgung am Lebensende im engen Zeitrahmen der letzten 48 oder 72 Stunden des Lebens ist das Ziel des „Liverpool Care Pathway for the Dying Patient“. Dieser Ansatz wurde gefördert, um das Hospiz-Modell der Versorgung sterbender Patienten in nicht spezialisierte Versorgungsstrukturen zu übertragen [22]. Im Sinne dieser Bedeutung kann Versorgung am Lebensende auch verstanden und umgesetzt werden als Betreuungsstandard für sterbende Patienten, die keiner palliativen Versorgung bedürfen.

- Abb. 1 verdeutlicht die Unterschiede zwischen der engen und erweiterten Definition von End-of-Life Care. Mit Hinsicht auf die unterschiedlichen Bedeutungen dieses Begriffs und das Ausmaß der Überschneidungen zwischen Versorgung am Lebensende und Palliativversorgung wird in diesem Papier auf End-of-life Care nicht ausführlicher eingegangen.

\subsection{Terminal Care}

Terminal Care ist ein älterer Fachbegriff, der für die umfassende Betreuung von Patienten mit fortgeschrittenen Tumorerkrankungen und eingeschränkter Lebenserwartung verwendet wurde. Neuere Definitionen der Palliativversorgung beschränken sich nicht auf Patienten mit begrenzter Lebenserwartung im Endstadium einer Erkrankung. Somit hat der Begriff „Terminal Care“ an Bedeutung verloren und sollte nicht mehr verwendet werden oder nur zur Beschreibung der Betreuung in der allerletzten Phase (einige wenige Tage) der Erkrankung.

\subsection{Entlastungspflege (Respite Care)}

Familienmitglieder und andere Personen in der Primärversorgung eines Palliativpatienten in der häuslichen Umgebung können unter der kontinuierlichen Belastung der Betreuung leiden. Entlastungspflege bietet diesen Patienten und deren Betreuern eine geplante oder ungeplante Entlastungspause [23].

Entlastungspflege kann durch Tageskliniken, stationäre Einrichtungen oder spezialisierte ambulante Palliativdienste angeboten werden.

\section{Philosophie der Palliativversorgung $\nabla$}

Trotz der Unterschiede zwischen den nationalen Konzepten der Palliativversorgung findet sich in der Literatur eine Reihe gemeinsamer Werte und Prinzipien, die von Initiativen der Hospizund Palliativversorgung befürwortet und anerkannt werden $[11,24]$.

In allen europäischen Ländern wird von Experten der Palliativversorgung eine Reihe gemeinsamer Grundwerte anerkannt. Diese beinhalten die Werte der Patientenautonomie und der Würde des Patienten, die Notwendigkeit einer individuellen Planung und Entscheidungsfindung sowie einen ganzheitlichen Ansatz.

\subsection{Autonomie}

In der Palliativversorgung wird jede Person als autonomes und einzigartiges Individuum anerkannt und respektiert. Die Betreuung wird nur durchgeführt, wenn der Patient und/oder die Familie bereit sind, sie anzunehmen. Idealerweise behält der Patient seine Selbstbestimmung bezüglich der Entscheidungshoheit über den Betreuungsort, die Behandlungsmöglichkeiten und den Zugang zu spezialisierter Palliativversorgung.

Patienten sollten darin bestärkt werden, Entscheidungen zu treffen, wenn sie dies wünschen. Dies erfordert die Weitergabe adäquater Informationen bezüglich Diagnose, Prognose, Behandlung und Betreuungsoptionen sowie aller anderer relevanter Betreuungsaspekte.

Ein stetiger Diskussionspunkt ist die ethische Herausforderung, wenn die Entscheidungsfähigkeit des Patienten nicht gegeben ist oder er keine Entscheidungen treffen möchte und die Bewertung von Informationen sowie die Entscheidungsfindung auf die Familie oder das Palliative-Care-Personal übertragen werden. Das Gleichgewicht zwischen Autonomie und schützender Betreuung scheint durch kulturelle Faktoren beeinflusst zu werden, da die Übertragung der Entscheidungsfindung vom Patienten auf die Betreuungspersonen in einigen Ländern häufiger berichtet wird. 


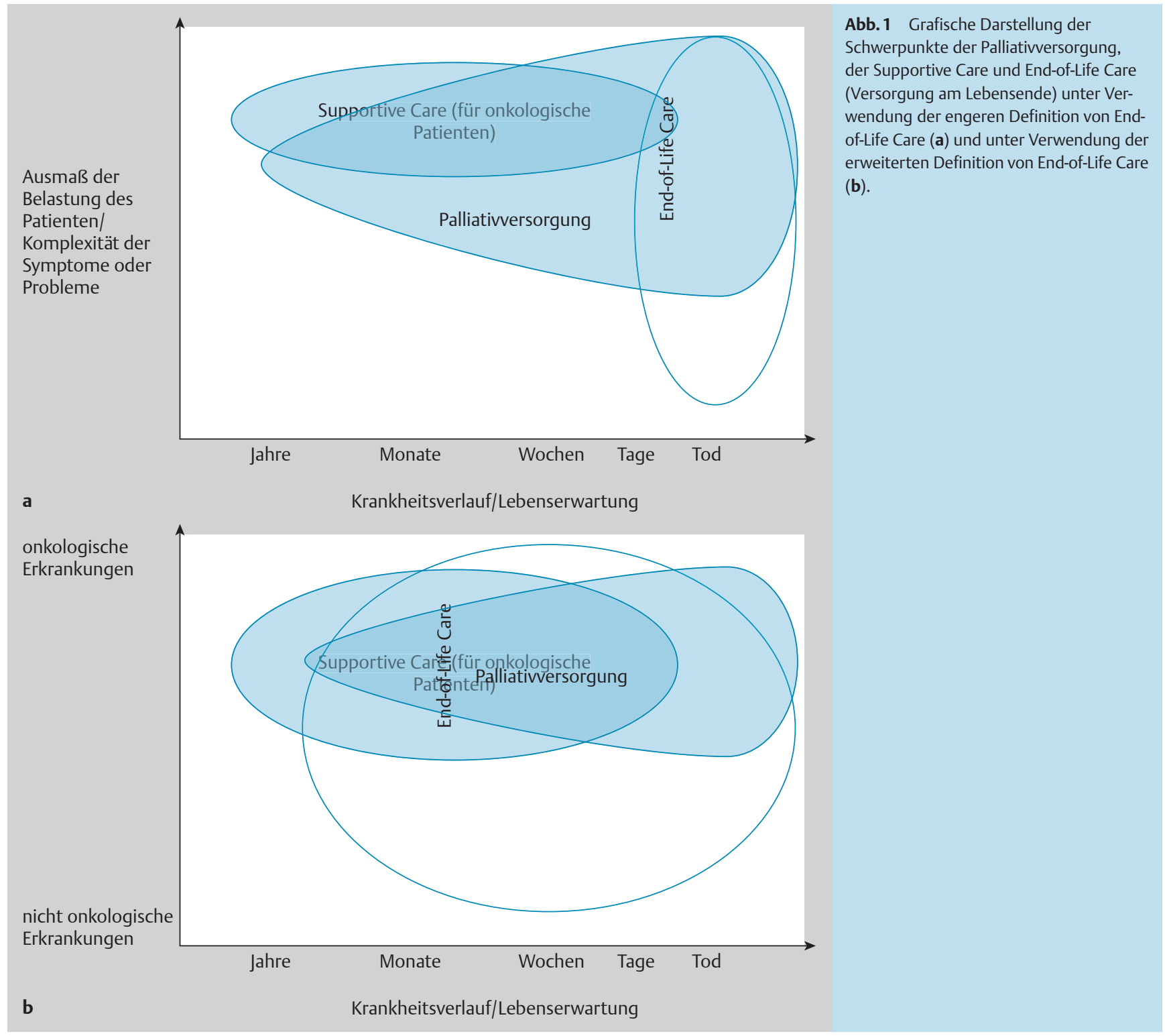

\subsection{Würde}

Palliativversorgung soll in einer respektvollen, offenen und sensiblen Weise durchgeführt werden, mit Einfühlungsvermögen sowohl gegenüber persönlichen, kulturellen und religiösen Werten, Glaubensinhalten und Gewohnheiten als auch gegenüber den Gesetzen jedes Landes.

Wie Lebensqualität scheint auch Würde ein Begriff zu sein, der für jeden Patienten eine individuelle Bedeutung hat mit unterschiedlichen Schwerpunkten und Prioritäten. Abhängig von der Definition kann Würde eher als eine inhärente Eigenschaft betrachtet werden, denn als Größe, die beschädigt werden oder verloren gehen kann. Unter diesem Aspekt ist die Palliativversorgung gefordert, Versorgungsstrukturen zu schaffen, in deren Rahmen Patienten ihre Würde erfahren und erleben können.

\subsection{Beziehung zwischen Patient und Versorgungsanbie-} ter

Die in der Palliativversorgung Tätigen sollten eine partnerschaftliche Beziehung mit den Patienten und Familien aufbauen. Patienten und Familien sind wichtige Partner bei der Planung der Betreuung und beim Management der Erkrankung.
Der salutogenetische Ansatz der Palliativversorgung legt den Schwerpunkt auf die Ressourcen und Kompetenzen der Patienten und nicht auf deren Probleme [11]. Dabei hat in jüngerer Zeit ein resilienzorientierter Ansatz zunehmend an Beachtung gewonnen. Resilienz beschreibt die Möglichkeit der Patienten, mit unheilbaren Erkrankungen und den damit verbundenen Problemen sowie dem durch die eingeschränkte Lebenserwartung bedingten Perspektivwechsel umzugehen. Das Konzept der Resilienz bietet einen Paradigmenwechsel an: Symptome, Risiken, Probleme und Verletzlichkeit zu fokussieren, ist defizitorientiert, während Resilienz den Fokus auf die Ressourcen des Patienten richtet. Resilienz unterstreicht die Bedeutung des öffentlichen Gesundheitswesens und schafft eine Partnerschaft zwischen Patienten, Versorgungspersonal und Gemeindestrukturen [25].

\subsection{Lebensqualität}

Ein zentrales Ziel der Palliativversorgung ist es, die bestmögliche Lebensqualität des Patienten zu erreichen, zu unterstützen und zu erhalten.

Die Betreuung ist geleitet von der Lebensqualität, die von jedem Menschen individuell definiert wird. Im Einzelnen wird zwi- 
schen biopsychosozialer und spiritueller Lebensqualität unterschieden, und es kommen standardisierte Fragebögen zur Erfassung gesundheitsbezogener und krankheitsspezifischer Lebensqualität zum Einsatz. Dennoch kann die Bedeutung von „Lebensqualität“" in der Palliativversorgung nur jeder Patient individuell für sich selbst definieren [11]. Wichtige Bereiche der individuellen Lebensqualität sowie Schwerpunkte innerhalb dieser Bereiche können sich häufig durch das Fortschreiten der Erkrankung ändern. Lebensqualität scheint eher von der wahrgenommenen Diskrepanz zwischen Erwartungen und tatsächlichen Möglichkeiten abhängig zu sein als von objektiven Einbußen der Leistungsfähigkeit.

\subsection{Einstellung gegenüber Leben und Tod}

Viele Definitionen von Palliative Care beinhalten eine Stellungnahme zu Leben und Tod [11]. Die erste Definition der WHO (World Health Organisation) von 1990 postulierte, dass „Palliativmedizin das Leben bejaht und das Sterben als normalen Prozess betrachtet", und andere Definitionen teilen diese Betrachtungsweise. Der Tod wird auf gleicher Ebene mit dem Leben gesehen, untrennbar davon und als Teil dessen.

Palliative Care versucht, den Tod weder zu beschleunigen noch ihn hinauszuschieben.

Euthanasie und ärztliche Beihilfe zum Suizid gehören nicht in den Bereich der Palliativversorgung [13].

„Der Wert des Lebens, der natürliche Prozess des Todes und die Tatsache, dass beide Möglichkeiten zu persönlichem Wachstum und zur Selbstverwirklichung darstellen“, sollten anerkannt werden [24].

\subsection{Kommunikation}

Gute Kommunikationsmöglichkeiten sind eine wichtige Voraussetzung für hochqualitative Palliativversorgung. Diese Kommunikation umfasst die Interaktion zwischen dem Patienten und dem Versorgungsteam, aber auch die Interaktion zwischen den Patienten und ihren Angehörigen sowie die Interaktionen zwischen verschiedenen Professionen und Versorgungsanbietern, die in die Betreuung involviert sind.

Unzufriedenheit und Beschwerden über die Betreuung sind häufiger auf ineffiziente Kommunikation als auf inadäquate Versorgungsleistung zurückzuführen [4], während eine effiziente Kommunikation die Betreuung des Patienten nachweislich verbessert [26]. Kommunikation in der Palliativversorgung ist viel mehr als ein reiner Austausch von Information. Schwierige und manchmal schmerzhafte Bereiche müssen Berücksichtigung finden, was Zeit, Verbindlichkeit und Aufrichtigkeit erfordert. Für das Personal in der Palliativversorgung kann es eine belastende Aufgabe sein, auf der einen Seite ehrliche und vollständige Informationen zu vermitteln und auf der anderen Seite die Hoffnungen der Patienten und deren Familien auf Überleben trotz des bevorstehenden Todes zu respektieren [27]. Für eine effiziente Kommunikation müssen angemessene Voraussetzungen geschaffen werden [4]; dies beinhaltet Übung und Ausbildung, angemessene und entsprechend ausgestattete Räumlichkeiten, Zeit zum Gespräch mit den Patienten und deren Familien sowie zum Austausch mit dem Team als auch den Zugang zu moderner Informationstechnologie.

\section{7 Öffentlichkeitsarbeit}

Der Europarat verkündete, dass es von äußerster Wichtigkeit ist, in den Mitgliedsstaaten der EU ein Klima der Akzeptanz für die Palliativversorgung zu schaffen [4]. Diesbezüglich ist es entschei- dend, gesellschaftliche Kapazitäten auszubauen und vorbeugende Gesundheitsvorsorge zu fördern, damit zukünftige Generationen dem Sterben und der Trauer, mit denen wir alle irgendwann konfrontiert werden, weniger ängstlich gegenüberstehen [25].

\subsection{Multiprofessioneller und interdisziplinärer Ansatz}

Teamarbeit wird als zentraler Bestandteil der Palliativversorgung angesehen [28]. Ein multiprofessionelles Team umfasst Mitglieder verschiedener Berufsgruppen und Fachgebiete des Gesundheitswesens und anderer Bereiche, die zusammenarbeiten, um die Betreuung der Patienten in einer bestimmten Situation sicherzustellen und/oder zu verbessern. Die Zusammensetzung des multiprofessionellen Teams hängt von verschiedenen Faktoren ab wie beispielsweise der zu versorgenden Patientengruppe, dem Ausmaß der zu leistenden Betreuung und den lokalen Gegebenheiten des jeweiligen Versorgungsbereichs [29].

Palliativversorgung sollte idealerweise in einem multiprofessionellen und interdisziplinären Rahmen angeboten werden. Wenngleich die palliative Grundversorgung durch eine Einzelperson einer bestimmten Berufsgruppe oder Disziplin geleistet werden kann, ist eine komplexe spezialisierte Palliativversorgung, die körperliche, psychische, soziale und spirituelle Unterstützung leisten kann (s. Abschnitt 5 „Ebenen der Palliativversorgung“), nur durch kontinuierliche Zusammenarbeit und Kommunikation der verschiedenen Professionen und Disziplinen zu gewährleisten.

Es gibt klare Anzeichen dafür, dass Teamarbeit in der Palliativversorgung von Vorteil für den Patienten ist [4]. Ein systematisches Literaturreview von Hearn und Higginson zu spezialisierten Palliativteams in der Onkologie hat gezeigt, dass diese Teams - verglichen mit der herkömmlichen Betreuung - die Patientenzufriedenheit verbessern und dass sie die Bedürfnisse des Patienten und der Familie besser erfassen und auch besser damit umgehen können [30].

Ferner hat sich gezeigt, dass mit einem multiprofessionellen Ansatz die Gesamtkosten der Betreuung aufgrund der geringeren stationären Verweildauer reduziert werden. Eine neuere systematische Literaturübersicht untermauert die Evidenz der positiven Wirkung der Palliativteams mit dem größten Effekt im Bereich der Schmerz- und Symptomkontrolle [31]. Die Erweiterung des Teams kann flexibel gestaltet werden und muss auf die Bedürfnisse des Patienten abgestimmt werden. Die Minimalversion kann somit aus einem niedergelassenen Hausarzt und einer spezialisierten Pflegefachkraft bestehen. Allerdings sind in den meisten Fällen Sozialarbeiter, Psychologen und/oder Ehrenamtliche mit eingebunden. Der Europarat empfiehlt, dass die Führungsrolle eines jeden Fachbereichs eines spezialisierten Palliativteams von einer speziell in Palliativversorgung qualifizierten Fachkraft besetzt sein sollte [4].

\subsection{Trauer und Trauerbegleitung}

Die Palliativversorgung bietet während der Erkrankung des Patienten den Familien und anderen ihnen nahestehenden, in die Betreuung eingebundenen Personen Unterstützung, hilft ihnen, sich auf den Verlust vorzubereiten und führt die Trauerbegleitung bei Bedarf nach dem Tod des Patienten fort. Angebote der Trauerbegleitung werden als ein zentraler Bestandteil einer palliativen Versorgung angesehen [4,5].

Das Erfassen und Abschätzen von Trauerreaktionen des Patienten und der Familie gehört zu jedem Zeitpunkt des Krankheitsverlaufs in einer an der Krankheitsentwicklung orientierten Weise zur Palliativbetreuung. Hierbei wird insbesondere auf Aspekte 


\begin{tabular}{|c|c|c|c|c|}
\hline & \multicolumn{4}{|c|}{ Palliativversorgung } \\
\hline & $\begin{array}{c}\text { palliativer } \\
\text { Versorgungsschutz }\end{array}$ & \multicolumn{2}{|c|}{$\begin{array}{l}\text { spezialisierte Unterstützung für } \\
\text { allgemeine Palliativversorgung }\end{array}$} & \begin{tabular}{|c|} 
spezialisierte \\
Palliativversorgung
\end{tabular} \\
\hline akute Versorgung & Krankenhaus & \multirow{4}{*}{$\begin{array}{l}\text { ehrenamtliche } \\
\text { Hospizdienste }\end{array}$} & \multirow{2}{*}{$\begin{array}{c}\text { palliativer } \\
\text { Konsildienst }\end{array}$} & \multirow{2}{*}{ Palliativstation } \\
\hline \multirow{2}{*}{ Langzeitversorgung } & Alten- und & & & \\
\hline & Pflegeheim & & \multirow[b]{2}{*}{$\begin{array}{c}\text { ambulante } \\
\text { Palliativteams }\end{array}$} & stationäres Hospiz \\
\hline Home Care & $\begin{array}{l}\text { niedergelassene } \\
\text { (Fach-)Ärzte } \\
\text { ambulante Kranken- } \\
\text { pflegedienste }\end{array}$ & & & Tageshospiz \\
\hline
\end{tabular}

Abb. 2 Bausteine der abgestuften Hospiz- und Palliativversorgung (Quelle: Nemeth C, Rottenhofer I. Abgestufte Hospizund Palliativversorgung in Österreich. Wien: Österreichisches Bundesinstitut für Gesundheitswesen; 2004). des Verlustes und der Trauer beim Erleben einer lebensbedrohlichen Erkrankung eingegangen. Nach dem Tod des Patienten werden der Familie Trauerbegleitung und weitere Unterstützung angeboten.

\section{Ebenen der Palliativversorgung \\ $\nabla$}

Palliativversorgung kann auf verschiedenen Ebenen stattfinden. Es sollten mindestens 2 Stufen angeboten werden: ein palliativer Versorgungsansatz und spezialisierte Palliativversorgung.

Gegenwärtig wird in den meisten Dokumenten ein 2-stufiges Modell beschrieben, und zwar wird unterschieden zwischen einem palliativen Versorgungsansatz und spezialisierter Palliativversorgung. Der palliative Versorgungsansatz wird von Nichtspezialisten angeboten, die nur gelegentlich Palliativpatienten behandeln. Im Gegensatz dazu wird spezialisierte Palliativversorgung erbracht von einem Team von entsprechend ausgebildeten Ärzten, Pflegefachkräften, Sozialarbeitern, Seelsorgern und weiteren ausgebildeten Personen, deren Expertise zur Optimierung der Lebensqualität von Menschen mit einer lebensbedrohenden oder belastenden chronischen Erkrankung erforderlich ist [5].

\subsection{Palliativer Versorgungsansatz}

Der palliative Versorgungsansatz ist ein Weg, um die Arbeitsweise und die Methoden der Palliativversorgung in nicht spezialisierte Strukturen zu integrieren. Dies schließt nicht nur pharmakologische und nichtpharmakologische Maßnahmen zur Symptomkontrolle ein, sondern auch die Kommunikation mit dem Patienten, der Familie und mit anderen Versorgungsanbietern sowie Entscheidungsfindungen und Zielsetzungen gemäß den Prinzipien der Palliativversorgung.

Der palliative Versorgungsansatz sollte niedergelassenen Ärzten und dem Personal in Krankenhäusern der Allgemeinversorgung wie auch ambulanten Pflegediensten und dem Personal in Pflegeheimen zugängig gemacht werden. Um diesen Versorgungsanbietern die Umsetzung des palliativen Versorgungsansatzes zu ermöglichen, muss die Palliativversorgung Bestandteil der Curricula der Grundausbildungen in Medizin, Pflege und anderen verwandten Berufsgruppen sein. Der Europarat empfiehlt, dass alle Berufsgruppen, die im Gesundheitswesen arbeiten, mit den Grundprinzipien der Palliativversorgung vertraut und in der Lage sein sollten, diese in die Praxis umzusetzen [4].

\subsection{Allgemeine Palliativversorgung}

Das beschriebene 2-stufige Modell kann auf 3 Stufen erweitert werden mit einem palliativen Versorgungsansatz, der allgemeinen und der spezialisierten Palliativversorgung. Allgemeine Palliativversorgung wird durch diejenigen Grundversorger und Spe- zialisten erbracht, die Patienten mit lebensbedrohlichen Krankheiten behandeln und über ein gutes Grundwissen und gute Basisfertigkeiten in Palliativversorgung verfügen.

Behandler, die häufiger in Palliativsituationen involviert sind, z. B. Onkologen oder Geriater, deren Haupttätigkeitsfeld jedoch nicht die Palliativversorgung ist, können eine spezialisierte Weiterbildung in Palliativversorgung erwerben und können dadurch eine zusätzliche Expertise anbieten. Diese Behandler können allgemeine Palliativversorgung leisten [4].

\subsection{Spezialisierte Palliativversorgung}

Patienten mit lebensbedrohlichen Erkrankungen und deren Angehörige können komplexe Bedürfnisse haben, die die Einbeziehung eines spezialisierten Palliativteams erfordern [29]. Spezialisierte Palliativversorgung beschreibt Versorgungsangebote, deren Schwerpunkt auf der Palliativversorgung liegt. Diese Dienste versorgen in der Regel Patienten mit komplexen und schwierigen Bedürfnissen, was einen höheren Ausbildungsgrad sowie eine intensivere Ausstattung mit Personal und anderen Ressourcen erfordert [4].

Spezialisierte Palliativversorgung wird von spezialisierten Versorgungsanbietern angeboten für Patienten, deren komplexe Anforderungen durch andere Behandlungsmöglichkeiten nicht hinreichend abgedeckt werden.

Alle Patienten mit einer fortschreitenden unheilbaren Erkrankung sollten Zugang zu einer spezialisierten Palliativversorgung haben, die sich proaktiv aller Symptome ihrer Erkrankung sowie der Auswirkung dieser Symptome auf die Patienten und deren Familien/Betreuer annimmt [29]. Patienten mit komplexen Bedürfnissen der palliativen Versorgung benötigen ein breites Spektrum an therapeutischen Interventionen zur Symptomkontrolle. Die zuständigen spezialisierten Palliativdienste müssen über entsprechend hochqualifiziertes Personal verfügen, um diese Interventionen sicher zu gewährleisten.

Spezialisierte Angebote der Palliativversorgung erfordern einen Teamansatz, der ein multiprofessionelles Team mit einer interdisziplinären Arbeitsweise kombiniert. Die Teammitglieder müssen hochqualifiziert sein und sollten ihren Tätigkeitsschwerpunkt in der Palliativversorgung haben.

Das Angebot spezialisierter Palliativversorgung muss von Fachkräften umgesetzt werden, die eine anerkannte Weiterbildung in spezialisierter Palliativversorgung absolviert haben $[5,29]$. Von den Teammitgliedern wird erwartet, dass sie Erfahrung im klinischen Management von Problemen aus vielen verschiedenen Bereichen haben, um den komplexen Bedürfnissen der Patienten gerecht werden zu können. Ihre Arbeit ist geprägt von einer substanziellen Expertise in der Betreuung von Patienten mit lebensbedrohlichen oder belastenden chronischen Erkrankungen und deren Familien. 
- Abb. 2 zeigt die Elemente des palliativen Versorgungsansatzes, der allgemeinen und der spezialisierten Palliativversorgung im Überblick.

\subsection{Palliative Kompetenzzentren (Centres of Excellence)} Das Modell kann noch um eine vierte Stufe erweitert werden, und zwar durch palliative Kompetenzzentren. Palliative Kompetenzzentren sollten spezialisierte Palliativversorgung in einer breiten Vielfalt von Settings anbieten, einschließlich stationärer und ambulanter Betreuung sowie fachlicher Beratung, und sie sollten darüber hinaus akademische Möglichkeiten zur Forschung sowie Aus-, Fort- und Weiterbildung vorhalten. Die Rolle der palliativen Kompetenzzentren wird noch diskutiert und ihre Position innerhalb des mehrstufigen Ansatzes der Palliativversorgung wird vom Ausgang dieser Diskussion abhängig sein.

Palliative Kompetenzzentren sollten Zentren für Aus-, Fort- und Weiterbildung, Forschung und Öffentlichkeitsarbeit sowie für die Entwicklung von Standards und neuen Methoden sein.

\section{Zielgruppen \\ $\nabla$}

\subsection{Patienten}

Palliativversorgung ist nicht auf vorab festgelegte medizinische Diagnosen beschränkt, sondern sollte für alle Patienten mit einer lebensbedrohenden Erkrankung zugänglich sein.

Palliativversorgung ist die passende Versorgung für alle Patienten und / oder Familien, die mit einer lebensbedrohlichen Erkrankung leben oder bei denen das Risiko einer lebensbedrohlichen Situation besteht, ungeachtet der Diagnose, der Prognose und des Alters, wenn sie unbefriedigte Erwartungen und/oder Bedürfnisse haben und bereit sind, die Betreuung zu akzeptieren [32]. Palliativversorgung kann die krankheitsmodifizierende Therapie ergänzen und unterstützen oder sie kann die alleinige Form der Betreuung werden. Einige Aspekte der Palliativversorgung können auch bei Patienten mit dem Risiko einer Erkrankung und deren Familien Anwendung finden.

Jedes Jahr sterben in Europa 1,6 Mio. Patienten an Krebserkrankungen und etwa 5,7 Mio. Menschen an anderen chronischen Erkrankungen.

In der europäischen Region (wie von der WHO definiert) sind 881 Mio. Einwohner registriert. Die altersstandardisierte Sterberate wurde für diese Region mit 9300 Todesfällen pro Mio. Einwohner berechnet. Von diesen sind ungefähr 1700 Todesfälle durch Krebserkrankungen und 6500 Todesfälle durch andere chronische Erkrankungen bedingt [33]. Diese Berechnung wird durch ein kürzlich veröffentlichtes Review gestützt, das besagt, dass es jedes Jahr 1,7 Mio. Todesfälle durch Krebserkrankungen in Europa gibt [34].

Die große Mehrzahl dieser Patienten leidet an Schmerzen, Atemnot und anderen körperlichen Symptomen oder benötigt Unterstützung bei psychosozialen oder spirituellen Problemen, die mit dem Fortschreiten ihrer Erkrankung auftreten können. Eine australische Arbeitsgruppe schätzte - unter Anwendung unterschiedlicher Methoden innerhalb eines konzeptionellen Rahmens - die Population, die Palliativversorgung benötigt, auf zwischen $50 \%$ und $89 \%$ aller sterbenden Patienten [35]. Während ein palliativer Versorgungsansatz den meisten dieser Patienten eine adäquate Betreuung bietet, wurde geschätzt, dass mindestens $20 \%$ der onkologischen Patienten und 5\% der nicht onkologischen Patienten in ihrem letzten Lebensjahr spezialisierte Palliativversorgung benötigen [36]. Diese Prozentsätze wurden im
Konsensprozess allerdings hinterfragt, und es wurden deutlich höhere Prozentzahlen sowohl von onkologischen als auch nicht onkologischen Patienten mit Bedarf an spezialisierter Palliativversorgung genannt. Die unterschiedlichen Schätzungen hängen jedoch auch von spezifischen Erkrankungsmustern in verschiedenen Ländern ab. Auch erstrecken sich die Behandlungszeiträume von wenigen Tagen bis zu mehreren Jahren. Mit den Fortschritten der modernen Medizin werden jedoch viele der betroffenen Patienten Palliativversorgung über einen längeren Zeitraum - und nicht nur im letzten Lebensjahr - benötigen.

Dies bedeutet, dass in der europäischen Region (gemäß WHODefinition) zu jedem Zeitpunkt mehr als 320000 onkologische und 285000 nicht onkologische Patienten Unterstützung oder Betreuung durch spezialisierte Palliativversorgung benötigen.

Diese Zahl ist eher vorsichtig geschätzt, da die Belastung durch Symptome und der Bedarf an Palliativversorgung häufig nicht erkannt werden und folglich möglicherweise viel höher sind als geschätzt. Auch gehen diese Schätzungen davon aus, dass die allgemeine Palliativversorgung gut entwickelt und generell verfügbar ist, was jedoch nur auf wenige europäische Länder zutrifft. Andere Berichte aus England gehen von höheren Mortalitätsraten durch onkologische und nicht onkologische Erkrankungen aus und schätzen den Prozentsatz an Patienten mit Bedarf an spezialisierter Palliativversorgung deutlich höher ein [37].

In allen europäischen Ländern kommt Palliativversorgung vorwiegend bei Patienten mit fortgeschrittenen Tumorerkrankungen zur Anwendung. Patienten mit anderen Erkrankungen wie neurologischen Erkrankungen, HIV / AIDS oder mit fortgeschrittenen Herz-, Lungen- oder Nierenerkrankungen können die gleichen palliativen Versorgungsbedürfnisse haben wie Tumorpatienten, der Zugang zur Palliativversorgung ist für sie jedoch ungleich schwerer.

Sowohl körperliche Symptome als auch psychologische, soziale und spirituelle Bedürfnisse kommen bei Patienten mit chronischen neurologischen, kardialen, zerebrovaskulären, pulmonalen oder nephrologischen Erkrankungen oder bei HIV/AIDS ebenso häufig vor wie bei Tumorerkrankungen. Das Muster der Symptome und der Krankheitsverlauf dieser Patienten können sich von denen eines Tumorpatienten unterscheiden [38,39], dennoch benötigen viele entweder einen palliativen Versorgungsansatz oder sogar spezialisierte Palliativversorgung, da die Intensität und Komplexität der Symptome und Probleme ähnlich ausgeprägt sein dürfte wie bei Tumorpatienten.

Der Zugang zu hochqualifizierter Palliativversorgung für nicht onkologische Patienten sollte im Rahmen der Entwicklung der nationalen und europäischen Gesundheitspolitik Priorität haben. Heute leiden mehr als 95\% der im Rahmen spezialisierter Palliativversorgungsangebote behandelten Patienten an Krebserkrankungen [40]. Der niedrige Prozentsatz an nicht onkologischen Patienten ist vor allem bedingt durch für sie höhere Barrieren beim Zugang zu Angeboten der Palliativversorgung, aber auch dadurch, dass Palliativversorgung in der Öffentlichkeit wie auch in der medizinischen Fachwelt häufig als ein auf onkologische Patienten beschränkter Ansatz wahrgenommen wird.

Ein adäquates Angebot von Palliativversorgung für nicht onkologische Patienten erfordert zusätzliche Ressourcen. Wenn nicht onkologische Patienten in gleichem Maße Zugang zur Palliativversorgung erhalten wie onkologische Patienten, werden die Prozentsätze von Patienten mit Bedarf an Palliativversorgung auf $40 \%$ (nicht onkologische Patienten) bzw. $60 \%$ (onkologische Patienten) geschätzt. 
Mit den demografischen Veränderungen der alternden Populationen wird sich das Muster der Mortalität ebenfalls verändern [41]. Immer mehr Menschen leben länger; der Anteil der über 60 -Jährigen hat deutlich zugenommen und wird in den nächsten 20 Jahren weiter anwachsen [41]. Bei zunehmender Lebenserwartung werden mehr Menschen an einer schweren chronischen Erkrankung, wie z.B. kardialen, zerebrovaskulären, pulmonalen Erkrankungen, sowie auch an onkologischen Erkrankungen sterben. Da mehr Menschen ein höheres Alter erreichen und chronische Krankheiten mit zunehmendem Lebensalter häufiger werden, wird der Anteil der Menschen, die mit einer solchen Erkrankung leben und daran leiden, ebenso größer werden. Mit zunehmendem medizinischem Fortschritt werden die Patienten mit chronischen und beeinträchtigenden Erkrankungen länger leben können und in der Folge über einen längeren Zeitraum Palliativversorgung benötigen.

Die Anzahl an onkologischen Patienten mit Bedarf an Palliativversorgung wird in den kommenden Jahren ebenfalls ansteigen, da zu erwarten ist, dass die Zahl der Patienten mit onkologischen Erkrankungen aufgrund der früheren Diagnosestellung, der verbesserten Behandlung und der längeren Überlebensdauer zunehmen wird [40].

Strategische Planungen im Gesundheitswesen erfordern Ansätze, die der zunehmenden Nachfrage Rechnung tragen können. Eine jüngere Analyse der Trends der Vergangenheit und Voraussagen für die Zukunft bezüglich Mortalität in England und Wales unterstreicht die Dringlichkeit, sich auf einen großen Zuwachs an alten und sterbenden Menschen einzustellen. Dies bedeutet, dass entweder deutlich mehr stationäre Betreuungsmöglichkeiten oder eine ausgeprägte Entwicklung der gemeindebasierten Betreuung erforderlich ist [42].

\subsection{Krankheitsstadien}

Es gibt keinen definierten Zeitpunkt im Verlauf einer Erkrankung für den Übergang von kurativer zu palliativer Betreuung.

Palliativversorgung ist die adäquate Versorgung für alle Patienten ab dem Zeitpunkt der Diagnose einer lebensbedrohlichen oder beeinträchtigenden Erkrankung [5]. Der Begriff „lebensbedrohliche oder beeinträchtigende Erkrankung“ wird hier verwendet, um eine Gruppe von Patienten aller Altersklassen und mit einem breiten Spektrum von Diagnosen zusammenzufassen, die mit einer andauernden oder rezidivierenden belastenden Erkrankung leben, die die Aktivitäten des täglichen Lebens einschränkt oder die Lebenserwartung vorhersehbar verkürzt.

Die meisten Patienten brauchen nur in weit fortgeschrittenen Krankheitsstadien Palliativversorgung, aber einige Patienten benötigen palliative Interventionen zur Überbrückung von Krisen bereits zu einem früheren Zeitpunkt ihres Krankheitsverlaufs. Dies kann einen Zeitraum von mehreren Jahren, Wochen oder Tagen betreffen [4].

Beim Übergang von kurativer zu palliativer Betreuung handelt es sich oft um keinen eindeutigen Zeitpunkt, sondern vielmehr um einen graduellen Prozess, in dessen Verlauf sich das Behandlungsziel mehr und mehr von der Lebensverlängerung um jeden Preis zur Erhaltung der Lebensqualität verschiebt, mit der Notwendigkeit, den Behandlungserfolg und die Nebenwirkungen sorgfältig gegeneinander abzuwägen.

\subsection{Kinder und Jugendliche}

Palliativversorgung für Kinder stellt einen speziellen - wenngleich eng mit der Palliativversorgung für Erwachsene verwandten - Fachbereich dar [43].
Der Europarat weist auf die spezifische Herausforderung der Palliativversorgung für Kinder mit unheilbaren und lebensbedrohlichen Erkrankungen hin [4].

Palliativversorgung für Kinder beginnt mit der Diagnosestellung und wird unabhängig davon, ob das Kind eine kurative Behandlung erhält oder nicht, fortgeführt [44]. Die Versorgungseinheit ist das Kind und seine Familie.

Eine lebenslimitierende Erkrankung ist bei Kindern definiert als eine Erkrankung, bei der ein vorzeitiger Tod üblich ist, wie z.B. bei der Muskeldystrophie Duchenne [44]. Lebensbedrohende Erkrankungen sind Krankheiten, die aufgrund ihrer Schwere mit einer hohen Wahrscheinlichkeit zu einem vorzeitigen Tod führen, bei denen jedoch auch die Chance des Langzeitüberlebens bis ins Erwachsenenalter besteht; dies betrifft z.B. Kinder, die eine Tumortherapie erhalten oder mit akuten schweren Verletzungen auf eine Intensivstation aufgenommen werden.

Pädiatrische Palliativversorgung ist im Vergleich zur Palliativversorgung für Erwachsene durch ein heterogeneres Spektrum an Erkrankungen und einen höheren Prozentsatz an nicht onkologischen Diagnosen charakterisiert. Palliativversorgung für Kinder und Jugendliche kann in 4 Kategorien eingeteilt werden, basierend auf einem Leitfaden zur Entwicklung von palliativen Versorgungsangeboten für Kinder, erarbeitet durch die Association for Children's Palliative Care (ACT) und das Royal College of Paediatrics and Child Health in England [45].

- Gruppe 1: lebensbedrohende Erkrankungen, für die kurative Therapien existieren, bei denen jedoch ein Therapieversagen wahrscheinlich ist. Zugang zu palliativer Versorgung kann in Kombination mit kurativen Therapien während der Phase eines unklaren Therapieerfolgs oder bei Therapieversagen notwendig werden.

- Gruppe 2: Erkrankungen wie z. B. zystische Fibrose ${ }^{3}$, bei denen ein vorzeitiger Tod unausweichlich ist und bei denen lang andauernde intensive Behandlungen zum Ziel haben, das Leben zu verlängern und die Teilnahme an normalen Aktivitäten zu ermöglichen.

- Gruppe 3: fortschreitende Erkrankungen ohne kurative Behandlungsmöglichkeiten, bei denen häufig über viele Jahre eine ausschließlich palliative Versorgung durchgeführt wird; z.B. Batten-Krankheit und Muskeldystrophie.

- Gruppe 4: irreversible, jedoch nicht progressive Erkrankungen mit komplexen Versorgungsbedürfnissen, die zu Komplikationen führen und mit der Wahrscheinlichkeit eines vorzeitigen Todes einhergehen. Beispiele sind schwere Zerebralparesen und schwere Mehrfachbehinderungen nach einer Hirn- oder Rückenmarkserkrankung.

Spezifische pädiatrische Angebote der Palliativversorgung zur stationären Behandlung und häuslichen Versorgung sollten aufgebaut werden. Für das Kind und seine Familie sollte ein umfassendes Spektrum an klinischen Versorgungsangeboten und Schulungsmöglichkeiten zur Verfügung stehen, und zwar in einer dem Alter sowie den kognitiven und schulischen Möglichkeiten angemessenen Form [44].

Das Leitfaden-Dokument zu Standards für die pädiatrische Palliativversorgung in Europa, erarbeitet durch das Lenkungskomitee

\footnotetext{
${ }^{3}$ Inwieweit Kinder mit einer zystischen Fibrose im Laufe ihrer Erkrankung einer Palliativversorgung bedürfen, wird kontrovers diskutiert. Aufgrund der verbesserten Therapiemöglichkeiten und der Option einer Lungentransplantation wird die zystische Fibrose heute von einigen Fachleuten nicht mehr zwangsläufig als lebenslimitierende Erkrankung betrachtet. Der Nutzen einer Lungentransplantation bei dieser Indikation ist jedoch empirisch umstritten [46].
} 
der EAPC Task Force für Palliativversorgung bei Kindern und Jugendlichen (IMPaCCT), zeigt auf, dass palliative Versorgungsangebote für Kinder und deren Familien unzureichend vorhanden sind [44]. Es wurde z. B. auf das Fehlen von gemeindenahen Ressourcen hingewiesen, die es den Familien ermöglichen würden, ihr Kind zu Hause zu betreuen, ebenso auf einen Mangel an Entlastungsangeboten.

Das Zuhause der Familie sollte, wann immer möglich, das Zentrum der Betreuung bleiben. Jede Familie sollte Zugang zu einem multiprofessionellen ganzheitlichen pädiatrischen Palliativ-CareTeam für die Betreuung zu Hause haben [44].

\section{Danksagungen}

Die Forschungstätigkeit wurde durch eine nicht zweckgebundene Zuwendung seitens der Klinik für Palliativmedizin der RWTH Aachen (Deutschland) unterstützt. Das Manuskript wurde durch Saskia Jünger vorbereitet. Wir möchten uns bei den Experten bedanken, die Zeit und Mühe für das kritische Gegenlesen des Manuskripts investiert haben: Franco De Conno, Carl-Johan Fürst, Geoffrey Hanks, Irene Higginson, Stein Kaasa, Phil Larkin und Friedemann Nauck. Wir möchten ebenfalls den Vorständen der nationalen Fachgesellschaften für ihre engagierte Teilnahme am Delphi-Verfahren danken. Ohne ihr Engagement und ihre Unterstützung wäre es nicht möglich gewesen, diese Empfehlungen in so kurzer Zeit zu entwickeln.

\section{Hinweis}

In diesem offiziellen Positionspapier der Europäischen Gesellschaft für Palliative Care (EAPC) umreißen und erklären Lukas Radbruch, Sheila Payne und die Vorstandsmitglieder der EAPC die Empfehlungen der Gesellschaft für eine gemeinsame Terminologie und gemeinsame Qualitätsrichtlinien.

\section{Literatur}

1 Centeno C, Clark D, Lynch T et al. Facts and indicators on palliative care development in 52 countries of the WHO European region: results of an EAPC task force. Palliat Med 2007; 21: 463-471

2 Centeno C, Clark D, Lynch T et al. EAPC Atlas of Palliative Care in Europe. Houston: IAHPC Press; 2007

3 Gunten CF von. Humpty-Dumpty Syndrome. Palliat Med 2007; 21: 461-462

4 Council of Europe. Recommendation Rec (2003) 24 of the Committee of Ministers to member states on the organisation of palliative care, 2003. Im Internet: http://www.coe.int/t/dg3/health/Source/Rec(2003) 24_en.pdf; Stand: 02.08.2011

5 National Consensus Project for Quality Palliative Care. Clinical Practice Guidelines for Quality Palliative Care, 2004. Pittsburgh: 2004

6 Lunder U, Sauter S, Fürst CF. Evidence-based palliative care: beliefs and evidence for changing practice. Palliat Med 2004; 18: 265-266

7 Tomlinson S. National Action Planning Workshop on End-of-life Care. Winnipeg: Health Canada Secretariat on Palliative and End-of-life Care; 2002

8 Department of Health. End of Life Care Strategy: Promoting high quality care for all adults at the end of life. London: DH; 2008; Im Internet: http://www.dh.gov.uk/en/Publicationsandstatistics/Publications/Pub licationsPolicyAndGuidance/DH_086277; Stand: 02.08.2011

9 National Consensus Project for Quality Palliative Care. Clinical Practice Guidelines for Quality Palliative Care,; 2nd edn. 2009; Im Internet: http://www.nationalconsensusproject.org/Guideline.pdf; Stand: 02.08.2011

10 Jaspers B, Schindler J. Stand der Palliativmedizin und Hospizarbeit in Deutschland und im Vergleich zu ausgewählten Staaten (Belgien, Frankreich, Großbritannien, Niederlande, Norwegen, Österreich, Polen, Schweden, Schweiz, Spanien). Berlin: Enquete-Kommission des Bundestages „Ethik und Recht der modernen Medizin“; 2005

11 Pastrana T, Jünger S, Ostgathe C et al. A matter of definition - key elements identified in a discourse analysis of definitions of palliative care. Palliat Med 2008; 22: 222-232
12 European Association for Palliative Care. Definition of Palliative Care, 1998; Im Internet: http://www.eapcnet.org/about/definition.html; Stand: 02.08.2011

13 Materstvedt LJ, Clark D, Ellershaw J et al. Euthanasia and physicianassisted suicide: a view from an EAPC Ethics Task Force. Palliat Med 2003; 17: 97-101; discussion 102-179

14 Sepúlveda C, Marlin A, Yoshida T et al. Palliative care: the World Health Organization's global perspective. J Pain Symptom Manage 2002; 24: 91-96

15 World Health Organization. Cancer pain relief and palliative care. Report of a WHO Expert Committee. Geneva: WHO; 1990

16 World Health Organization. National Cancer Control Programmes. Policies and managerial guidelines, 2nd ed. Geneva: WHO; 2002

17 Help the Hospices. Definition of hospice care. Im Internet: http://www. helpthehospices.org.uk/about-hospice-care/what-is-hospice-care/; Stand: 02.08.2011

18 International Association for Hospice and Palliative Care. Definition of Hospice/Palliative Care. Im Internet: http://www.hospicecare.com/Or ganisation/\#Definition; Stand: 02.08.2011

19 Multinational Association of Supportive Care in Cancer. MASCC strategic plan, 2007; Im Internet: http://www.mascc.org; Stand: 02.08.2011

20 Smyth JF. Disclosing gaps between supportive and palliative care: the past 20 years. Support Care Cancer 2008; 16: 109-111

21 Radbruch L, Nauck F, Fuchs M et al. What is palliative care in Germany? Results from a representative survey. J Pain Symptom Manage 2002; 23: 471-483

22 Gambles M, Stirzaker S, Jack BA et al. The Liverpool Care Pathway in hospices: an exploratory study of doctor and nurse perceptions. Int J Palliat Nurs 2006; 12: 414-421

23 Ingleton $C$, Payne S, Nolan $M$ et al. Respite in palliative care: a review and discussion of the literature. Palliat Med 2003; 17: 567-575

24 Lunder $U$. Identifying effective ways of implementation of palliative care into Slovene health care system. Research Paper. Budapest: Center for Policy Studies, Open Society Institute; 2005

25 Monroe B, Oliviere D, eds. Resilience in palliative care: achievement in adversity, 1st edn. Oxford: Oxford University Press; 2007

26 Effective Health Care. Informing, communication and sharing decisions with people who have cancer. University of York: NHS Centre for Reviews and Dissemination; 2000

27 National Institute for Health and Clinical Excellence. Improving supportive and palliative care for adults with cancer. The manual. 2004; Im Internet: www.nice.org.uk/nicemedia/pdf/csgspmanual.pdf; Stand: 02.08.2011

28 Jünger S, Pestinger M, Elsner $F$ et al. Criteria for successful multiprofessional cooperation in palliative care teams. Palliat Med 2007; 21: $347-$ 354

29 Clinical Standards Board for Scotland. Clinical Standards for Specialist Palliative Care. Edinburgh: NHS Scotland; 2002

30 Hearn J, Higginson IJ. Do specialist palliative care teams improve outcomes for cancer patients? A systematic literature review. Palliative Medicine 1998; 12: 317-332

31 Higginson IJ, Finlay IG, Goodwin DM et al. Is there evidence that palliative care teams alter end-of-life experiences of patients and their caregivers? J Pain Symptom Manage 2003; 25: 150-168

32 Ferris FD, Balfour HM, Bowen Ket al. A model to guide patient and family care: based on nationally accepted principles and norms of practice. J Pain Symptom Manage 2002; 24: 106-123

33 World Health Organization Regional Office for Europe. Mortality indicators by 67 causes of death, age and sex (HFA-MDB), 2007; Im Internet (updated July 2011): http://www.euro.who.int/en/what-we-do/dataand-evidence/databases/mortality-indicators-by-67-causes-of-death, -age-and-sex-hfa-mdb; Stand: 02.08.2011

34 Higginson IJ, Costantini $M$. Dying with cancer, living well with advanced cancer. Eur J Cancer 2008; 44: 1414-1424

35 McNamara B, Rosenwax $L K$, Holman $C D$. A method for defining and estimating the palliative care population. J Pain Symptom Manage 2006; 32: $5-12$

36 Ministerium für Gesundheit, Soziales, Frauen und Familie des Landes Nordrhein-Westfalen. Rahmenprogramm zur flächendeckenden Umsetzung der ambulanten palliativmedizinischen und palliativpflegerischen Versorgung in Nordrhein-Westfalen. Kooperatives integriertes Versorgungskonzept, 2005; http://www.mags.nrw.de/08_PDF/002/ konzeptpalliativ.pdf, Stand; 08.09.2009

37 Higginson I. Palliative and terminal care. Health care needs assessment, 2nd series. Oxford: Radcliffe Medical Press; 1997 
38 Lynn J, Adamson DM. Living well at the end of life: adapting health care to serious chronic illness in old age. Arlington, VA: Rand Health Cooperation; 2003

39 Murray SA, Kendall M, Boyd $K$ et al. Illness trajectories and palliative care. BMJ 2005; 330: 1007-1011

40 Ireland National Advisory Committee. Report of the National Advisory Committee on Palliative Care. Department of Health and Children; 2001

41 Davies E, Higginson IJ eds. Better Palliative Care for Older People. Copenhagen: World Health Organization Regional Office for Europe; 2004

42 Gomes B, Higginson IJ. Factors influencing death at home in terminally ill patients with cancer: systematic review. BMJ 2006; 332: 515-521
43 World Health Organization. WHO Definition of Palliative Care. Im Internet: http://www.who.int/cancer/palliative/definition/en/; Stand: 02.08.2011

44 Craig F, Abu-Saad Huijer H, Benini F et al. Steering Committee of the EAPC Task Force on Palliative Care for Children and Adolescents. IMPaCCT: standards for paediatric palliative care in Europe. Eur J Pall Care 2007; 14: 109-114

45 Association for Children's Palliative Care (ACT), Royal College of Paediatrics and Child Health. A guide to the development of children's palliative care services. Bristol, London: ACT; 1997

46 Liou TG, Adler FR, Cox DR et al. Lung Transplantation and Survival in Children with Cystic Fibrosis. NEJM 2007; 357: 2143-2152 